\section{MS30-P5 Ruthenium quinazoline complexes}

Petra Kuzman ${ }^{1}$, Anton Meden ${ }^{1}$, Bogdan Štefane

1. Faculty of Chemistry and Chemical Technology, University of Ljubljana, Večna pot 113, SI-1000 Ljubljana, Slovenia

email: pkuzman1@gmail.com

Our research is based on the synthesis, characterization and evaluation of ruthenium quinazoline coordination compounds. We have successfully synthesized quinazoline derivatives $\mathbf{4}$ that were further used as ligands for the synthesis of ruthenium complexes $\mathbf{5}$ (Scheme 1). Successfully synthesized ruthenium complexes were fully characterized using X-ray single crystal analysis. A detailed kinetic studies of $\mathrm{C}-\mathrm{H}$ activation reaction were carried out. Cyclometallation of ligands by transition metals is a very important reaction since it enables the synthesis of organometallic complexes with a carbon-metal bond. The most commonly used methods for the synthesis of cyclometallated complexes are (a) transmetallation (transfer of ligands from one metal to another) and (b) metallation with selective $\mathrm{C}-\mathrm{H}$ activation. 1,2 Organometallic reagents that are used in transmetallation reaction are expensive and often commercially unavailable. $\mathrm{C}-\mathrm{H}$ activation allows the selective and direct functionalization of the non-reactive (hetero)aromatic C-H bonds, which leads to economically and ecologically friendly synthesis. ${ }^{3}$

Keywords: Organometallic complexes, ruthenium, X-ray crystalography

\section{MS30-P6 Near-infrared to visible light-upconversion in molecular coordination complexes}

Laure Guenee ${ }^{1}$, Davood Zare ${ }^{2}$, Yan Suffren ${ }^{3}$, Andreas Hauser ${ }^{3}$, Claude Piguet $^{2}$

1. Laboratory of Crystallography, University of Geneva, 24 quai Ernest Ensermet, 1211 Geneva 4, Switzerland

2. Department of Inorganic and Analytical Chemistry, University of Geneva, 30 quai Ernest Ansermet, 1211 Geneva 4, Switzerland 3. Department of Physical Chemistry, University of Geneva, 30 quai Ernest Ansermet, 1211 Geneva 4, Switzerland

email: laure.guenee@unige.ch

Light-upconversion via stepwise energy transfer from a sensitizer to an activator exploits linear optics for converting low-energy infrared or near-infrared incident photons to higher energy emission. This phenomenom could be of potential use in solar cell technology [1] and for in situ biological probes and sensor [2]. Generally, this light-upconversion approach is restricted to activators possessing intermediate long-lived excited states such as those found for trivalent lanthanide cations dispersed in solid-state matrices. When the activator is embedded in a molecular complex, efficient nonradiative relaxation processes usually reduce excited state lifetimes to such an extent that upconversion becomes too inefficient to be detected under practical excitation intensities. However, with the development of the supramolecular chemistry of lanthanide complexes, using specially designed ligands, assemblies can be made where the geometry of the metal centre is controlled. We present here studies of crystallographic and photophysical properties on a series of isostructural trinuclear dimetallic helicates $\left[\mathrm{MLnM}(\mathrm{L} 1)_{3}\right]\left(\mathrm{CF}_{3} \mathrm{SO}_{3}\right)_{9}$ with $\mathrm{M}=\mathrm{Ga}(\mathrm{III})$ or $\mathrm{Cr}(\mathrm{III})$ and $\mathrm{Ln}=\mathrm{Y}(\mathrm{III})$ or $\mathrm{Er}(\mathrm{III})^{\prime}$, leading to a detailed rational analysis of the energy transfer upconversion (ETU) mechanism. A novel upconversion pathway is presented and we demonstrate that strong-field trivalent chromium chromophores irradiated with near-infrared photons produce upconverted green erbium-centered emission in discrete polynuclear triple-stranded helicates [3]. [1] Huang, X.; Han, S.; Huang, W.; Liu, X. Chem. Soc. Rev. 2013, 42, 173-201. [2] Liu, Y.; Tu, D.; Zhu, H.; Chen, X. Chem. Soc. Rev. 2013, 42, 6924-6958. [3] (a) Aboshyan-Sorgho, L.; Besnard, C.; Pattison, P.; Kittilstved, K. R.; Aebischer, A.; Bünzli, J.-C. G.; Hauser, A.; Piguet, C. Angew. Chem., Int. Ed. 2011, 50, 4108-4112. (b) Aboshyan-Sorgho, L.; Nozary, H.; Aebischer, A.; Bünzli, J.-C. G.; Morgantini, P.-Y.; Kittilstved, K. R.; Hauser, A.; Eliseeva, S. V.; Petoud, S.; Piguet, C. Am. Chem. Soc. 2012, 134, 12675-12684 and references therein. (c) Y. Suffren, D. Zare, S. V. Eliseeva, L. Guénée, H. Nozary, T. Lathion, L. Aboshyan-Sorgho, S. Petoud, A. Hauser and C. Piguet, J. Phys. Chem. C, 2013, 117, 26957-26963. (d) Y. Suffren, D. Zare, L. Guénée, S. V. Eliseeva, H. Nozary, L. Aboshyan-Sorgho, S. Petoud, A. Hauser and C. Piguet, Dalton Trans., 2015, 44, 2529-2540.

Keywords: Light-upconversion, lanthanide complexes 